Article

\title{
A Deletion in GDF7 is Associated with a Heritable Forebrain Commissural Malformation Concurrent with Ventriculomegaly and Interhemispheric Cysts in Cats
}

\author{
Yoshihiko Yu ${ }^{1,2,+}$, Erica K. Creighton ${ }^{1, \dagger}$, Reuben M. Buckley ${ }^{1}$, Leslie A. Lyons ${ }^{1, *}$ \\ and 99 Lives Consortium $\ddagger$ \\ 1 Department of Veterinary Medicine and Surgery, College of Veterinary Medicine, University of Missouri, \\ Columbia, MO 65211, USA; yoshi.yu@nvlu.ac.jp (Y.Y.); erica-creighton@idexx.com (E.K.C.); \\ buckleyrm@missouri.edu (R.M.B.) \\ 2 Laboratory of Veterinary Radiology, Nippon Veterinary and Life Science University, Musashino, \\ Tokyo 180-8602, Japan \\ * Correspondence: lyonsla@missouri.edu \\ + The authors contributed equally to this work. \\ $\ddagger$ Membership of the 99 Lives Consortium is provided in the Acknowledgments.
}

Received: 15 May 2020; Accepted: 16 June 2020; Published: 19 June 2020

\begin{abstract}
An inherited neurologic syndrome in a family of mixed-breed Oriental cats has been characterized as forebrain commissural malformation, concurrent with ventriculomegaly and interhemispheric cysts. However, the genetic basis for this autosomal recessive syndrome in cats is unknown. Forty-three cats were genotyped on the Illumina Infinium Feline 63K iSelect DNA Array and used for analyses. Genome-wide association studies, including a sib-transmission disequilibrium test and a case-control association analysis, and homozygosity mapping, identified a critical region on cat chromosome A3. Short-read whole genome sequencing was completed for a cat trio segregating with the syndrome. A homozygous $7 \mathrm{bp}$ deletion in growth differentiation factor 7 (GDF7) (c.221_227delGCCGCGC [p.Arg74Profs]) was identified in affected cats, by comparison to the 99 Lives Cat variant dataset, validated using Sanger sequencing and genotyped by fragment analyses. This variant was not identified in 192 unaffected cats in the 99 Lives dataset. The variant segregated concordantly in an extended pedigree. In mice, GDF7 mRNA is expressed within the roof plate when commissural axons initiate ventrally-directed growth. This finding emphasized the importance of GDF7 in the neurodevelopmental process in the mammalian brain. A genetic test can be developed for use by cat breeders to eradicate this variant.
\end{abstract}

Keywords: feline; Felis catus; brain malformation; BMP12; neurodevelopment; genetics; genomics; mendelian traits; genome-wide association study; whole genome sequencing

\section{Introduction}

Congenital brain malformations in humans are caused by genetic variants, in utero infection, or other environmental factors. Dogs and cats are also occasionally diagnosed with congenital brain malformations (reviewed in [1]), which are noted as breed predispositions, familial aggregations, or sporadic cases, especially in dogs [2-6]. Congenital hydrocephalus is common in toy and brachycephalic dog breeds, such as the Maltese, Yorkshire terrier, Chihuahua, toy poodle and pug dogs [7]. Widespread in Cavalier King Charles Spaniels, Chiari-like malformation is a common cause of foramen magnum obstruction, and results in the secondary syringomyelia in dogs, characterized by the mismatch of size between the brain and the skull [8]. 
Similarly, high grades of brachycephaly in cats are also associated with malformations of the calvarial and facial bones, as well as dental malformations or respiratory abnormalities [9-12]. A familial craniofacial malformation with meningoencephalocele has been recognized in Burmese cats [13], which is caused by ALX Homeobox 1 (ALX1) variant [14]. However, feline brain malformations with (suspected) idiopathic nature are mostly reported as sporadic events [15-20]. Overall, the genetic factors contributing to brain (mal) formation and structural congenital brain disease in dogs and cats are largely unknown.

In an effort to develop a breed of cats having similar phenotypes to a tiger, including a small rounded ear, a mixed breed cat derived from the Oriental cat breed was discovered to have small rounded ears and hence, was used as a foundation sire for a breeding program. Outcross and backcross breeding indicated the phenotype was autosomal recessive [21]. However, a magnetic resonance imaging (MRI) examination of a kitten with the desired ear phenotype, which had an accidental head injury from a fall, indicated the presence of congenital hydrocephalus. Additional MRIs of the breeding stock suggested cats with the ear phenotype had congenital brain malformations. These cats have small rounded ear pinnae and doming of the head (Figure 1). This extended family of mixed-breed cats derived from the Oriental breed has been characterized clinically and histopathologically with forebrain commissural malformation concurrent with ventriculomegaly and interhemispheric cysts [21]. The forebrain malformations include dysgenesis of the septum pellucidum, interthalamic adhesion, and all the midline commissures, excluding the rostral white commissure, as well as hippocampal hypoplasia. Clinical symptoms include mild generalized ataxia when walking, and mild to marked postural reaction deficits, although cranial nerve examination and segmental reflexes are within normal limits. All the cats with neurological signs have midline and limbic structure abnormalities, dilated ventricles and hemispheral cysts with or without a suprapineal cyst. These findings resemble a mild variant of holoprosencephaly (HPE) in human (OMIM: 236,100 and others). Although variations in the severity of the forebrain commissural malformation were seen, most affected cats are hydrocephalic. No chromosomal abnormalities are noted in a karyotypic analysis of the cats. Segregation analysis suggests an autosomal recessive mode of inheritance; however, the causal variant remained unknown [21].

As a result of the potentially harmful impacts associated with the trait, the breeder promptly discontinued the breeding program and altered subsequent cats. However, some carriers for the trait had already been adopted for other breeding programs. A group of affected cats were presented to the researchers for pathological and genetic studies. Sample collection from the cats in the owner's breeding program and cats from controlled breeding within the university colony supported the genetic investigation of the abnormal brain development and mode of inheritance.

Genome-wide association studies (GWAS), using a sib-transmission disequilibrium test (sib-TDT) and a case-control analysis, and homozygosity mapping were conducted to detect an associated genomic region for the syndrome using genotypes from a feline single nucleotide polymorphism (SNP) DNA array [22]. Whole genome sequencing (WGS) was conducted on a cat trio segregating for the syndrome to define the location and identify candidate variants.

\section{Materials and Methods}

\subsection{Sampling and Pedigree}

All procedures were performed with an approved University of Missouri (MU) Institutional Animal Care and Use Committee protocol (ACUC protocol \# 8292). Four affected and two carrier cats were donated and housed at the MU colony for controlled breeding. Additional buccal swab and cadaver samples from an external breeding program were provided voluntarily by the breeder/owner $(N=129)$. DNA samples were extracted using DNeasy Blood \& Tissue Kit (Qiagen, Valencia, CA, USA). The quality of the DNA samples was visualized and confirmed by agarose gel electrophoresis. DNA samples whose concentration was insufficient were whole genome amplified, using the REPLI-g Mini Kit (Qiagen). The relationship of the ascertained cats was confirmed using short tandem repeat 
(STR) markers, as previously described [23]. Parentage analysis was performed using the computer program COLONY [24,25]. Clinical and histopathological features of the syndrome were characterized previously [21]. Although some cats were phenotyped based on MRI and/or histopathology, most cats were assumed to have the brain malformation based on the ear morphology, since clinically healthy cats had elongated (normal) ears and clinically affected cats had the small, rounded ear type [21] (Figure 1). Images or cadavers of cats were not always available.

(a)

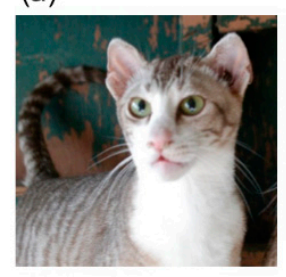

(d)

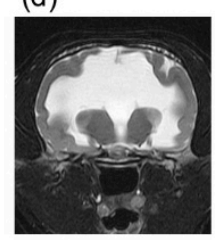

(b)

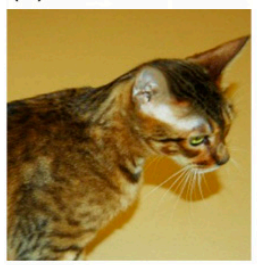

(c)

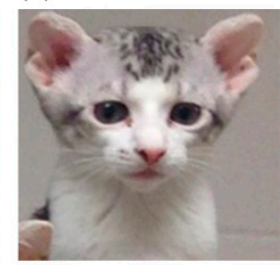

(g) (e)

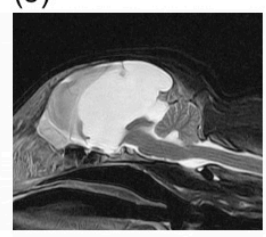

(f)

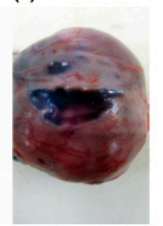

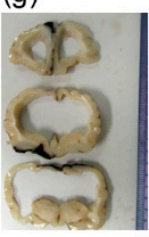

Figure 1. Domestic cats with heritable forebrain commissural malformation. Note the abnormal presentation of the pinnae used to determine affection status. (a) Frank-affected sire (left). (b) Camilla-carrier dam. (c) Bobble-affected offspring. These three cats $(\mathbf{a}-\mathbf{c})$ were whole genome sequenced. (d) Transverse plane of T2-weighted magnetic resonance imaging of an affected cat at the level of the thalamus. Severe ventriculomegaly, thinning of the cerebral parenchyma and midline structure deficits are seen. A part of the parietal lobe is deficient. (e) Mid-sagittal plane of T2-weighted magnetic resonance imaging of an affected cat (the same cat as (d)). Midline structure deficits are recognized. Note that the spinal cord is formed normally. Interhemispheric cysts are also seen at the rostrotentorial region and the quadrigeminal cistern. Due to the presence of cysts, cerebellar herniation is seen. (f) Gross dorsal view of the dissected head at necropsy. The skin was removed, and the skull was exposed. (g) Transverse sections of formalin-fixed brain tissue at the level of frontal lobe and thalamus. Severe ventriculomegaly, thinning of the cerebral parenchyma and midline structure deficits are seen. Note that a cat whose magnetic resonance imaging of (d) and (e) are presented here is different from cats whose gross pathological pictures of $(\mathbf{f})$ and $(\mathbf{g})$ are provided here.

\subsection{DNA Array Genotyping}

Fifty-two genomic DNA samples ( 600 ng each) were submitted to GeneSeek (Neogene, Lincoln, NE, USA) for SNP genotyping on the Illumina Infinium Feline 63K iSelect DNA Array (Illumina, San Diego, CA, USA) [22]. The original SNP positions were based on an early assembly of the cat genome [26], and have been since relocalized to the latest feline genome assembly, Felis_catus_9.0. The SNP positions based on the Felis_catus_9.0 assembly were used for the analyses and the required map file is available. [27]. Quality control of the SNP data was performed using PLINK (v1.07) [28]. The following criteria were applied: (i) individuals with genotyping success rate of $<80 \%$ were removed (-mind 0.2); (ii) SNP markers with a genotyping rate $<80 \%$ were removed (-geno 0.2 ); and (iii) SNPs with a minor allele frequency of 0.05 or less were removed (-maf 0.05$)$. Furthermore, SNPs that were previously reported to have missing $\geq 10 \%$ of genotypes and Mendelian errors [22], and that remained after quality controls were excluded.

\subsection{Genome-Wide Association Studies}

After the SNP pruning described above, GWAS were conducted using PLINK. Sib-TDT [29] was performed using the DFAM procedure in PLINK (-dfam). This method implements sib-TDT and also 
includes unrelated individuals in the analysis. A case-control association analysis was performed (-assoc). The genomic inflation factor was calculated using the function (-adjust). Multi-dimensional scaling (MDS) analysis was conducted (-genome) and MDS plots were generated to visualize the population stratification, using PLINK and R software (version 3.3.3; R Foundation for Statistical Computing, Vienna, Austria), respectively. A quantile-quantile (QQ) plot was created using R. Genome-wide significance for both analyses, which was determined using 100,000 permutations (-mperm 100000). Manhattan plots from the sib-TDT, case-control association and permutation analyses were generated using R. The MDS plot was used to reselect cats to minimize stratification between cases and controls for the secondary case-control association analysis, by visual interpretation.

\subsection{Haplotype Analysis}

An approximately $6 \mathrm{Mb}$ region surrounding highly associated SNPs was extracted, including 81 SNPs, from SNP chrA3.163737349 at chromosome position A3: 123,014,546 to SNP chrA3.156620632 at chromosome position A3: 128,837,125. The haplotype boundaries were visually confirmed using Haploview (version 4.2) [30]. Linkage disequilibrium (LD) blocks were identified using the solid spine of LD method in Haploview. Haplotype sequences are estimated using an accelerated EM algorithm, as implemented in Haploview. When analyzing LD blocks and haplotypes, SNPs with MAF of 0\% were allowed and included, because most cases showed the consistent genotypes at each SNP.

\subsection{Homozygosity Analysis}

Homozygosity analysis was performed using PLINK. SNPs within a $1000 \mathrm{~kb}$ window, containing at least 25, were investigated for runs of homozygosity (-homozyg-window-kb 1000,-homozyg-snp 25). In each window, five missing genotypes (20\%) and a single heterozygote ( $2 \%)$ were tolerated (-homozyg-window-missing 5, -homozyg-window-het 1). The threshold of homozygosity match was set as 0.99 (-homozyg-match 0.99). A homozygous block was characterized by five SNPs ( 200-250 kb). Consensus homozygosity blocks were identified as overlaps between individual homozygosity blocks (-consensus-match, -homozyg-group).

\subsection{Whole Genome Sequencing}

A trio of cats including an affected sire, a carrier dam and an affected offspring was selected for WGS as part of the 99 Lives Cat Genome Sequencing Initiative (http://felinegenetics.missouri.edu/99lives). These cats were produced at the MU colony; thus, the parentage was known. DNA extraction and library preparation were conducted as previously described [31]. A minimum of $4 \mu \mathrm{g}$ genomic DNA was submitted for WGS to the MU DNA Core Facility. Two PCR-free libraries with insertion sizes of $350 \mathrm{bp}$ and $550 \mathrm{bp}$ were constructed for each cat using the TruSeq DNA PCR Free library preparation kit (Illumina). The Illumina HiSeq 2000 (Illumina) was used to generate sequence data.

Sequence reads were mapped to the latest feline genome assembly, Felis_catus_9.0, and processed as previously described [27]. Briefly, read mapping was conducted with Burrows-Wheeler Aligner (BWA) version 0.7.17 [32]. Duplicates were marked using Picard tool MarkDuplicates (http://broadinstitute. github.io/picard/). Potential insertions or deletions (indels) realignment was performed using the Genome Analysis Tool Kit (GATK version 3.8) [33] IndelRealigner. Variants were called using GATK HaplotypeCaller in gVCF mode [34]. VarSeq v2.0.2 (Golden Helix, Bozeman, MT, USA) was used to annotate variants with Ensembl 99 gene annotations and identify variants unique to the trio cats and absent from 192 unaffected unrelated domestic cats. Exonic variants were extracted from the dataset, including variants $21 \mathrm{bp}$ flanking the exons to ensure inclusion of variants that may affect splice donor and accept sites. Candidate variants segregating across the trio were visualized using Integrative Genomics Viewer (IGV) [35]. 


\subsection{Variant Validation and Genotyping}

PCR and Sanger sequencing were performed to validate the 7 bp deletion in the candidate gene GDF7 for cats that were submitted to WGS. The primer sequences were: forward primer: 5'-AGCGACATCATGAACTGGTG-3' ${ }^{\prime}$, reverse primer: 5' -CCACGGAGCCCATGGACC-3' . PCR was performed using AccuPrime GC-Rich DNA Polymerase (Invitrogen, Carlsbad, CA, USA). PCR was performed following the manufacturer's instructions, with the annealing temperature of $61^{\circ} \mathrm{C}$ and 35 cycles. PCR amplicon was purified using QIAquick Gel Extraction Kit (Qiagen), or using ExoSAP-IT PCR Product Cleanup Reagent (Thermo Fisher Scientific, Waltham, MA, USA). Sanger sequencing was conducted at the MU DNA Core Facility using an Applied Biosystems 3730xl DNA Analyzer (Applied Biosystems, Foster City, CA, USA) with BigDye Terminator v3.1 Cycle Sequencing Kit (Applied Biosystems).

Fragment analysis was conducted for population screening. PCR conditions and reagents used were the same as above, except the forward primer was fluorescein amidite [FAM] labeled at the $5^{\prime}$ end. Fragment analysis was conducted at the MU DNA Core Facility using an Applied Biosystems 3730xl DNA Analyzer (Applied Biosystems). The expected wildtype fragment size was $294 \mathrm{bp}$, while the mutant fragment size was expected as $287 \mathrm{bp}$. Amplicons were analyzed using STRand software [36].

\section{Results}

\subsection{Pedigree and Genotyping}

Using 18 STRs, the parentage for 69 of 129 cats was determined with a high likelihood using the COLONY software [24,25] (data not shown), producing a pedigree of 79 cats (Figure S1). For GWAS, 52 cats were selected using owner provided and pedigree information, including 26 cases, and 26 controls, in which 43 cats were included in the pedigree (Figure S1). Cat DNA samples were genotyped on Feline 63K SNP array (File S1). Selection criteria for genotyping focused on cats that were as unrelated as possible. Nine cats with call rates below $80 \%$ were removed, and 478 SNPs were removed with missingness rates $>20 \%$. An additional 22,297 SNPs were also removed with minor allele frequencies $<0.05$. After filtering, 20 cases and 23 controls remained with a genotyping rate of 0.977 across 40,263 SNPs. Furthermore, 372 SNPs were excluded, due to missing $\geq 10 \%$ of genotypes and Mendelian errors previously reported [22]. The GWAS was conducted with 39,891 SNPs.

\subsection{Association Studies}

Sib-TDT was conducted on the pedigree formed by the 20 cases and 23 controls. After permutation testing, no SNPs were significant; however, nine SNPs with the highest, the second-highest, or the third-highest association were localized to cat chromosome A3:123,055,238-128,667,138 on the Felis_catus_9.0, extending approximately $5.6 \mathrm{Mb}$ (Table 1). The result of the sib-TDT analysis was presented as a Manhattan plot (Figure 2a). In the initial case-control association analysis, 65 SNPs had genome-wide significance and were located cat chromosome A3: 116,714,934-129,668,450, extending $\sim 13.0 \mathrm{Mb}$ and $\mathrm{C} 1:$ 105,429,018-115,412,315, extending $\sim 10.0 \mathrm{Mb}$ (Table 1). However, the genomic inflation factor was 1.89; thus, the MDS plot (Figure S2) was used to reselect cases and controls for the analysis. A second case-control association analysis was performed with 14 cases and nine controls, and the genomic inflation factor was reduced to one. Seventeen SNPs showed genome-wide significance and were located cat chromosome A3: 119,105,247-129,372,537, encompassing $\sim 10.3 \mathrm{Mb}$ (Figure 2b, Table 1). This chromosome A3 region encompassed the entire region suggested by the sib-TDT, and was within the initial case-control association analysis. 
Table 1. Single nucleotide polymorphism (SNP) associations for cats with heritable forebrain commissural malformation in the sib-transmission disequilibrium test (sib-TDT) and case-control association analyses.

\begin{tabular}{cccccc}
\hline SNP & Chr & Position $^{+}$ & Sib-TDT & Case-Control (Initial) & Case-Control (2nd) \\
\hline chrA3.164724433 & A3 & 123353491 & $>0.6$ & 0.0001 & 0.0045 \\
chrA3.164567500 & A3 & 122318611 & $>0.6$ & 0.0001 & 0.0045 \\
chrA3.164340161 & A3 & 122513677 & $>0.6$ & 0.0001 & 0.0045 \\
chrA3.164113252 & A3 & 122698750 & $>0.6$ & 0.0001 & 0.0045 \\
chrA3.163320257 & A3 & 123353491 & 0.5639 & 0.0001 & 0.0045 \\
chrA3.162970354 & A3 & 123644765 & $>0.6$ & 0.0001 & 0.0045 \\
chrA3.162343840 & A3 & 124176474 & $>0.6$ & 0.0004 & 0.0045 \\
chrA3.158624618 & A3 & 127189752 & $>0.6$ & 0.0001 & 0.0045 \\
chrA3.159621145 & A3 & 126377299 & 0.5639 & 0.0001 & 0.0082 \\
chrA3.162413594 & A3 & 124100380 & $>0.6$ & 0.0014 & 0.0137 \\
chrA3.156826206 & A3 & 128667138 & 0.5639 & 0.0001 & 0.0169 \\
chrA3.156620632 & A3 & 128837125 & $>0.6$ & 0.0001 & 0.0169 \\
chrA3.155936886 & A3 & 129372537 & $>0.6$ & 0.0004 & 0.0169 \\
chrA3.168960567 & A3 & 119105247 & $>0.6$ & 0.0128 & 0.0261 \\
chrA3.168031908 & A3 & 119810207 & $>0.6$ & 0.0264 & 0.0261 \\
chrA3.167492986 & A3 & 120088757 & $>0.6$ & 0.0061 & 0.0261 \\
chrA3.167322483 & A3 & 120215597 & $>0.6$ & 0.0292 & 0.0261 \\
chrA3.162621987 & A3 & 123934341 & 0.5521 & 0.0014 & $>0.05$ \\
chrA3.163679766 & A3 & 123055238 & 0.5639 & 0.0004 & $>0.05$ \\
chrA3.161984351 & A3 & 124475589 & 0.5639 & 0.0002 & $>0.05$ \\
chrA3.161943004 & A3 & 124509146 & 0.5639 & 0.0002 & $>0.05$ \\
chrA3.161399869 & A3 & 124945294 & 0.5639 & 0.0020 & $>0.05$ \\
chrA3.160673309 & A3 & 125511595 & 0.5639 & 0.0002 & $>0.05$ \\
\hline
\end{tabular}

$p$-values were presented with up to four decimal places. * SNP IDs are based on an early cat genome assembly [26]

${ }^{+}$Positions based on current cat genome assembly [27].

(a)

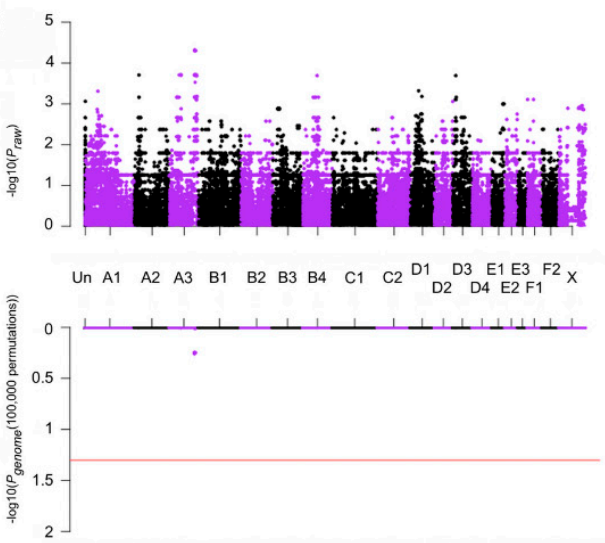

(b)

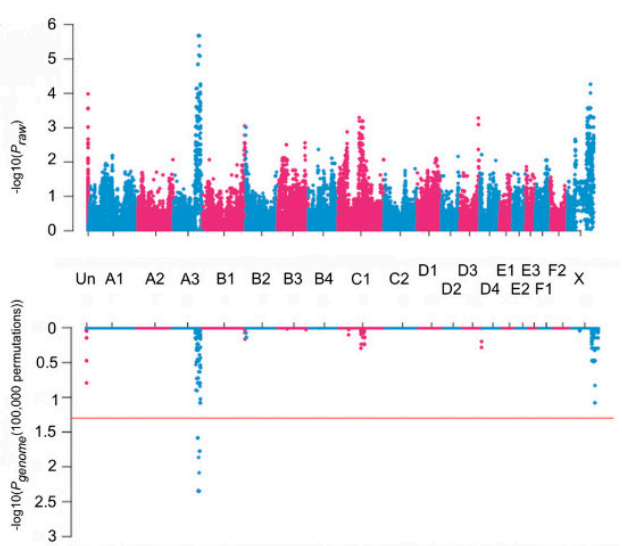

Figure 2. Manhattan plot of the genome-wide association studies (GWAS) for heritable forebrain commissural malformation in cats. Cats (20 cases and 23 controls) were genotyped on the Illumina Infinium Feline 63K iSelect DNA Array (Illumina, San Diego, CA, USA) and used for GWAS. In both panels of (a) and (b), the upper plots exhibit the $P_{\text {raw }}$ value of the analysis, while the lower exhibits the $P_{\text {genome }}$ values after 100,000 permutations. Red horizontal lines indicate genome-wide significance $\left(P_{\text {genome }}=0.05,-\log _{10}=1.3\right)$. (a) Sib-TDT analysis. Genome-wide significance was not achieved. (b) Case-control association analysis. Significant association is localized to chromosome A3 for 17 SNPs. The genomic inflation was 1 .

\subsection{Haplotype Analysis}

The $6 \mathrm{Mb}$ region, on chrA3: from approximately 123 to $129 \mathrm{Mb}$ and encompassing the overlapped region identified in GWAS, was visually inspected for common haplotypes using Haploview. In affected cats, a large extended LD block encompassing approximately $4.3 \mathrm{Mb}$ (A3: 123,082,369-127,348,216) was 
identified with a $95 \%$ frequency of the sequential haplotype. Considering that two cats had $82.7 \%$ and $91.4 \%$ genotyping rate, one cat had $98.8 \%$ and the others had $100 \%$ genotyping rate in this area, a few missing produced the remaining haplotypes (File S2). Short and discontinuous LD blocks are identified by Haploview in controls. There are various haplotype sequences and frequencies approximately within the $6 \mathrm{Mb}$ regions in unaffected cats.

\subsection{Homozygosity Analysis}

Homozygosity mapping was performed on 20 cases and 23 controls. The homozygosity analysis identified the same location on chromosome A3 in 18 of 20 affected cats, excluding the same two cases that did not have sufficiently high genotyping rates, with A3: 125,601,560-127,684,693, spanning approximately $2.1 \mathrm{Mb}$, and no unaffected cats were homozygosity (Table S1). The region was identified by the two genome-wide association analyses (Table 1). Although other ROHs were identified, none were specific to cases or as extensive.

\subsection{Whole Genome Sequencing}

Cat genomes have been submitted to the NCBI short read archive under BioProject: PRJNA528515; Accessions PRJNA343385; SRX2654400 (Sire), SRX2654398 (dam) and SRX2654399 (offspring). Genome sequence analyses and variant calling for the 99 Lives project has been previously described [37]. Approximately 2.5 million variants were ascertained across 195 cats in the exonic portion of the dataset, which included $21 \mathrm{bp}$ of exon flank sequence. No candidate genes were identified on cat chromosome A3 during the initial analysis, when considering the sire and offspring to be homozygous affected, and considering the dam as an obligate carrier for an alternative allele (Table 2). Only an intergenic variant $(\mathrm{C} 1: 106,990,675)$ and an intronic variant in sperm antigen with calponin homology and coiled-coil domains (SPECC1) (E1:9,973,078) met the segregation criteria. Using relaxed constraints, where affected cats were allowed to also be considered as carriers, four more variants were identified $(C 1: 96,095,693, C 1: 96,839,645$ and D2:33,368,378) with only one variant located within the critical region and also in a gene coding region (Table 2). This variant was a 7 bp deletion in the coding region of GDF7 (c.221_227delGCCGCGC [p.Arg74Profs*17]) at the position A3:127002233 (ENSFCAT00000063603). The variant was identified as homozygous in the affected sire, heterozygous in the obligate carrier dam, heterozygous in the affected offspring, and absent from the other 192 domestic cats. Although each cat in the trio had an average of $\sim 30 \times$ genome coverage, the sire had $18 \times$ coverage within the region, the dam had $\sim 14 \times$ coverage with seven reads per allele, and the affected offspring had $\sim 16 \times$ coverage, with only one of the reads representing the reference allele, likely misrepresenting the offspring as heterozygous, and visual inspection with IGV suggested the affected offspring was instead very likely homozygous for the variant (Figure 3). The affected cat was confirmed as a homozygote for the alternate allele by genotyping. The GDF7 variant was predicted to cause a truncated protein with 89 amino acids, while the wildtype protein has 455 amino acids (Figure S3). Feline GDF7 amino acid sequence is predicted to be $86.2 \%, 90.1 \%, 84.6 \%, 77.8 \%$ and $77.2 \%$ identical to human, horse, cow, rat and mouse, respectively (Figure S3). In addition, comparison of the GDF7 locus between the Felis_catus_9.0 and Felis_catus_8.0 genome assemblies, revealed the region containing the GDF7 candidate variant is absent from the Felis_catus_8.0 assembly, indicating the importance of the updated reference genome for trait discovery. 
Table 2. Variants identified in 99 Lives whole genome sequence dataset considering segregation within the trio.

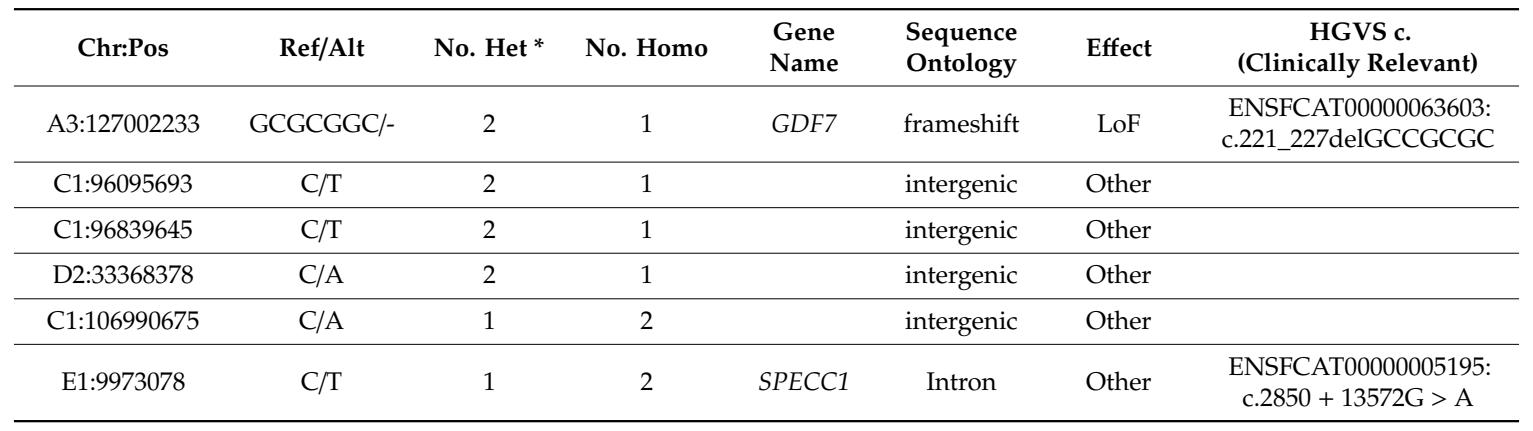

* Only the dam should be heterozygous for the variant in the dataset and the sire and offspring homozygous for the variant. Sequence data was poor within the critical region on cat Chromosome A3, and the affected offspring was erroneously considered heterozygous by the Genome Analysis Tool Kit (GATK version 3.8). Four variants are identified when the offspring is considered heterozygous.

\section{(a) Affected Sire}

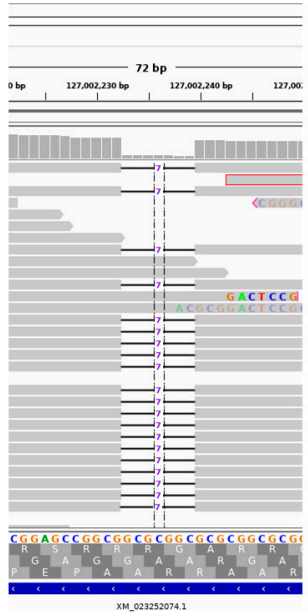

\section{(b) Carrier Dam}

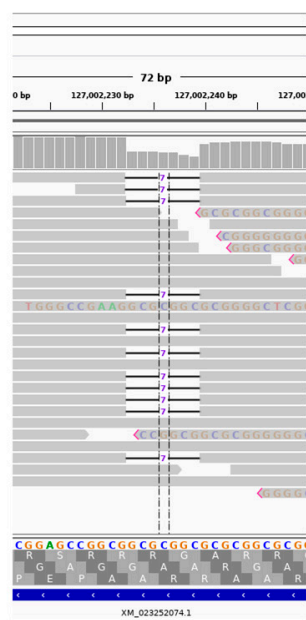

(c)

Affected

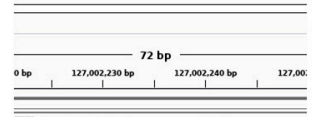

Offspring

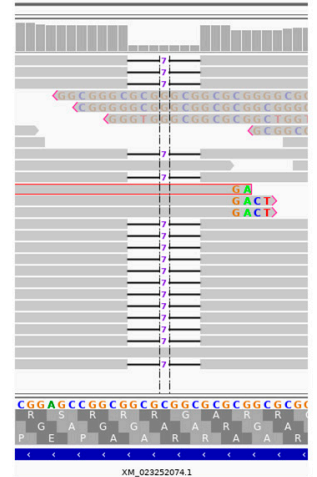

Figure 3. Depiction of the whole genome sequence reads using the Integrated Genome Viewer (IGV) of (a) affected sire, (b) carrier dam, and (c) affected offspring for the GDF7 variant. Grey horizontal bars represent individual reads, while grey vertical bars at the top of each sub figure represent depth of coverage. Notice in affected individuals $(\mathbf{a}, \mathbf{c})$, coverage is close to zero in the deleted region, while, in the carrier sequencing (b), coverage is approximately $50 \%$. Additionally, in affected individuals (a,c), reads with high numbers of mismatches are indicative of the misidentification of an indel, and tend to occur near the ends of reads. 


\subsection{Variant Validation and Genotyping}

Sanger sequencing was performed to confirm the identified GDF7 c.221_227delGCCGCGC in affected and obligate carrier cats, including the cats in the WGS trio. The 7 bp deletion in GDF7 was screened in 25 affected, 39 unaffected, and two cats with unknown phenotype in the extended pedigree using fragment analysis (Figure S4). Both unknown cats were homozygous for the variant allele. Overall, 13 of 14 suspected wildtype cats in the extended pedigree were concordant, and one cat genotyped as a heterozygote. Of 25 suspected carriers, 23 genotyped as heterozygote and two as wildtype normal. Of 22 suspected affected cats, 20 genotyped as homozygous for the variant, one as heterozygous and one as wildtype normal.

\section{Discussion}

Brain malformations are occasionally identified in veterinary practice. However, little is known about the genetic causes and interactions for brain malformation. Due to the health concerns associated with breed development, particularly in dog breeds [38,39], many breeders have become more vigilant to health-associated consequences of selection based on morphological phenotypes. Feline brain malformation syndrome seen in this extended family happened to be generated in the course of breeding selection for the ear morphological phenotype.

Most of the cat samples had been archived as frozen cadavers by the breeder, and later provided to the researchers. As a result of poor documentation of relationships and disease status, a pedigree was established by determining parentage using STRs, age, and gender of the cats and from interviews with the breeder. Ear phenotypes, which were used as a proxy for disease, were difficult to determine from frozen cadavers. Due to the significant inbreeding and backcrossing required to maintain the phenotype, 18 STRs were often insufficient to determine parentage. However, some known breedings were available from the university colony. Overall, an extended pedigree was developed, and was expected to be sufficient for GWAS and WGS investigations for the causal variant. Furthermore, a variant dataset from WGS of domestic cats, the 99 Lives Cat Genome Sequencing Initiative, which has revealed the causative variants for several cat diseases and traits in the last several years [31,40-46], was considered to facilitate the variant filtering to find the private variants.

In humans, HPE is the most common malformation of the prosencephalon, and its prevalence is approximate 1 in 10,000 births [47]. A common feature of HPE includes the incomplete separation of the anterior part of the forebrain or telencephalon. The previous study indicated this feline heritable brain malformation syndrome resembled a mild form of HPE [21]. Many genes have been reported to cause HPE in humans (reviewed in [48-50]). However, GDF7, also known as bone morphogenetic protein 12 (BMP12), has not been reported to be associated with HPE in humans. Initially, GDF7 activity was shown to be required for the specification of neuronal identity in the spinal cord [51]. GDF7 mRNA is expressed within the roof plate, when commissural axons initiate to grow ventrally-directed. Furthermore, GDF7-null mutant mice show hydrocephalus, and they show considerable variation in the location of the dilated ventricle [51]. This evidence supports these findings that the frameshift mutation in GDF7 causing the truncated protein is highly likely to be associated with this heritable brain malformation syndrome in cats. Transcriptomic and proteomic analyses would be essential to ascertain that this GDF7 variant causes heritable forebrain commissural malformation in cats.

The variable severity of this syndrome in the cat pedigree was reported previously [21]. In humans, heterogeneity in familial HPE is also identified even if different individuals are carrying the same mutation [52-54]. The influence of environmental or teratogenic factors or modifier genes have been suggested for the spectrum (reviewed in $[47,48,50]$ ). Assuming no exposure to teratogen and relatively homogeneous living environment, the presence of modifier genes is suspected for the variable severity of the dilated ventricles and supratentorial cysts in cats presented here.

Bone morphogenetic proteins (BMPs) belong to the transforming growth factor- $\beta$ (TGF- $\beta$ ) superfamily of proteins that are involved in many functions such as cell proliferation, differentiation, apoptosis, cell fate determination and morphogenesis [55]. The BMPs also play various roles in the 
neural development [56]. Among them, GDF7 also known as BMP12, plays an essential role in bone and cartilage formation as well [57]. Except for hydrocephalus seen in GDF7-null mutant mice [51], several phenotypes caused by GDF7 deficient mice have been reported, including the subtle effect on Achilles tendon [58], increased endochondral bone growth [59], seminal vesicle defects and sterility [60], and smaller bone cross-sectional geometric parameters [61]. In addition, a variant in GDF7 (rs3072) has been reported to increase risk for Barrett's esophagus and esophageal adenocarcinoma [62,63]. Although, to the authors' knowledge, there was no report about the involvement of GDF7 in ear or skull morphology, there is a possibility that small rounded pinnae and/or domed craniums may be influenced by the GDF7 variant, because GDF7, also known as BMP12, has been considered to play a negative role on chondrogenesis [59], and to be involved in the structural integrity of bone [61].

In conclusion, the combination of GWAS, homozygosity mapping and WGS identified a $7 \mathrm{bp}$ deletion in GDF7 (c.221_227delGCCGCGC), which is the most likely variant causing feline forebrain commissural malformation, concurrent with ventriculomegaly and interhemispheric cysts in this domestic cat lineage, although the functional analysis has not been achieved to prove the deterministic mechanism. Furthermore, this study highlights the importance of GDF7 in the neurodevelopmental course in cats, and brings new insight into neurodevelopmental biology. Cat breeders can now perform a genetic test to eradicate the GDF7 mutation from the breeding population.

Supplementary Materials: The following are available online at http://www.mdpi.com/2073-4425/11/6/672/s1. Table S1: Regions of homozygosity was unique to 18 cats with the inherited forebrain commissural malformation, and were absent in all the unaffected cats. Figure S1: Pedigree of cats segregating for an autosomal recessive forebrain commissural malformation. Relationships of 79 cats ( 27 nuclear families) provided by the breeder and confirmed with genetic testing of short tandem repeats when possible. Arrow indicates the proband. Circles indicate females, squares indicate males, and diamonds indicate unknown sex. Filled symbols represent cats with small rounded ears, which were suspected to have forebrain commissural malformation concurrent with ventriculomegaly and interhemispheric cysts. Half-filled represent obligate carriers. Symbols with question marks represent cats with unknown phenotype. A symbol with no fill indicates the cat is known to be completely unrelated and not expected to be a carrier. The cats genotyped on the DNA array and used for genome-wide association studies and homozygosity mapping are indicated by a " $\mathrm{T}$ " on the upper left of the symbol (The nine cats removed by quality control are not indicated). A black filled circle at the left bottom of symbol are individuals that were whole genome sequenced. Cats with a bar above the symbol were confirmed by magnetic resonance imaging. Cats with an open circle to the upper right had histology performed at necropsy. The cats' ID/name is indicated below the symbol. Size in basepairs of the genotypes for the $7 \mathrm{bp}$ GDF7 indel are indicated below each cat available. Figure S2: Multi-dimensional scaling plot and quantile-quantile plot of cases and controls for genome-association analyses. (a) Multi-dimensional scaling (MDS) plot of cats used for the initial case-control association analysis. The genomic inflation was 1.89. Therefore, cats clustered within the blue rectangular area were selected for the second case-control association analysis as visual inspection suggests less stratification between cases and controls. The genomic inflation factor was reduced to 1 . $(b, c)$ The quantile-quantile plots of cats used for the initial (b) and second (c) analyses demonstrate the observed versus expected-log(p) values. Figure S3: Protein sequence alignment of GDF7 in cats (Felis catus) and other species. GDF7 protein sequences are aligned from wildtype cat (Felis catus), GDF7 mutant cat, cow (Bos Taurus: NP_001193030.1 [ARS-UCD1.2]), horse (Equus caballus: XP_023475218.1 [EquCab3.0]), mouse (Mus musculus: NP_001299805.1 [GRCm38.p4]), and rat (Rattus norvegicus: XP_006239940.1 [Rnor_6.0]). Identical amino acids to those of Felis catus sequence are represented as a $\operatorname{dot}($.$) . Deleted amino acids are represented as a dash (-). A 7 \mathrm{bp}$ deletion causes a frameshift and changes the amino acid sequence from 74th position (highlighted in yellow), starting with an arginine to a proline change, which results in the truncated protein with a stop codon 17 amino acids downstream. Figure S4. Variant validation by Sanger sequencing and fragment analysis. (a) Sanger sequence of a wildtype and homozygous affected cat for the $7 \mathrm{bp}$ GDF7 variant (boxed region). (b) Fluorescence-based fragment analysis using an ABI 3730XL for the GDF7 variant. Left-homozygous wildtype with 294 bp fragment, middle-heterozygous with 287 and 294 bp fragments, and right-affected with $287 \mathrm{bp}$ fragment. LIZ standard (Applied Biosystems, Foster City, CA, USA) was used to size DNA fragments. File S1: Ped file for PLINK of cats genotyped using Illumina Infinium Feline 63K iSelect DNA Array. File S2: SNPs $(n=81)$ forming common haplotype for cats in the association studies.

Author Contributions: Conceptualization, L.A.L.; Methodology, L.A.L.; Software, R.M.B., Y.Y.; Validation, Y.Y.; Formal Analysis, E.K.C., Y.Y.; Investigation, E.K.C., Y.Y., R.M.B.; Resources, L.A.L., E.K.C.; Data Curation, L.A.L., R.M.B.; Writing-Original Draft Preparation, Y.Y., E.K.C.; Writing-Review \& Editing, Y.Y., R.M.B., E.K.C., L.A.L.; Visualization, Y.Y., R.M.B., E.K.C.; Supervision, L.A.L.; Project Administration, L.A.L.; Funding Acquisition, L.A.L. All authors have read and agreed to the published version of the manuscript.

Funding: This study was supported by in part by NIH Office of Research Infrastructure Programs (OD R24OD01092), Winn Feline Foundation (MT-13-010), the Cat Health Network (D14FE-552) and the MU Gilbreath McLorn Endowment for Comparative Medicine (L.A.L.). The authors thank the JSPS Overseas Challenge 
Program for Young Researchers (2017-2018) for sponsoring the visiting scholarship (Y.Y.) and the financial support from Mars, Inc. (R.M.B.).

Acknowledgments: We also thank Barbara Gandolfi and Thomas R. Juba for technical assistance and assistance with figures from Karen Clifford. 99 Lives Consortium (2019 cat analysis-99Lives195) Organizer: Leslie A. Lyons ${ }^{1}$; Data analyst: Reuben M. Buckley ${ }^{1}$; Each member of the 99 Lives Consortium (2019 cat analysis - 99Lives195) has provided at least one $>15 x$ coverage genome of the domestic cat or a wild felid to support the analyses of the dataset. Members: Reuben M. Buckley ${ }^{1}$, Danielle Aberdein ${ }^{2}$, Paulo C. Alves 3,4, Gregory S. Barsh 5,6, Rebecca R. Bellone 7, Tomas F. Bergström 8, Adam R. Boyko 9, Jeffrey A. Brockman 10, Margret L. Casal 11, Marta G. Castelhano ${ }^{12}$, Ottmar Distl ${ }^{13}$, Nicholas H. Dodman ${ }^{14}$, N. Matthew Ellinwood ${ }^{15}$, Jonathan E. Fogle ${ }^{16}$, Oliver P. Forman ${ }^{17}$, Dorian J. Garrick 2,15 , Edward I. Ginns ${ }^{18}$, Jens Häggström 19, Robert J. Harvey 20, Daisuke Hasegawa ${ }^{21}$, Bianca Haase 22, Christopher R. Helps ${ }^{23}$, Isabel Hernandez 24, Marjo K. Hytönen 25, Maria Kaukonen 25, Christopher B. Kaelin 5,6, Tomoki Kosho 26, Emilie Leclerc 27, Teri L. Lear ${ }^{28}$, Tosso Leeb ${ }^{29}$, Ronald H.L. Li ${ }^{30}$, Hannes Lohi 25, Maria Longeri ${ }^{31}$, Mark A. Magnuson 32, Richard Malik 33, Shrinivasrao P. Mane 34, John S. Munday 2, William J. Murphy ${ }^{35}$, Niels C. Pedersen 36, Simon M. Peterson-Jones 37, Max F. Rothschild 15, Clare Rusbridge 38, Beth Shapiro ${ }^{39}$, Joshua A. Stern ${ }^{36}$, William F. Swanson ${ }^{40}$, Karen A. Terio ${ }^{41}$, Rory J. Todhunter ${ }^{12}$, Wesley C. Warren ${ }^{42}$, Elizabeth A. Wilcox ${ }^{12}$, Julia H. Wildschutte ${ }^{43}$, Yoshihiko Yu ${ }^{21}$, Leslie A. Lyons ${ }^{1}$.

1 Department of Veterinary Medicine and Surgery, College of Veterinary Medicine, University of Missouri, Columbia, MO 65211, USA

2 School of Veterinary Science, Massey University, Palmerston North 4442, New Zealand

3 CIBIO/InBIO, Centro de Investigação em Biodiversidade e Recursos Genéticos/InBIO Associate Lab \& Faculdade de Ciências, Universidade do Porto, Campus e Vairão, 4485-661 Vila do Conde, Portugal Wildlife Biology Program, University of Montana, Missoula, MT 59812, USA

HudsonAlpha Institute for Biotechnology, Huntsville, AL 35806, USA

Department of Genetics, Stanford University, Stanford, CA 94305 USA

Veterinary Genetics Laboratory, University of California, Davis, CA 95616, USA

8 Department of Animal Breeding and Genetics, Swedish University of Agricultural Sciences, 75007 Uppsala, Sweden

9 Department of Biomedical Sciences, College of Veterinary Medicine, Cornell University, Ithaca, NY 14853, USA

10 Hill's Pet Nutrition Inc., Topeka, KS 66601, USA

11 Reproduction, and Pediatrics, School of Veterinary Medicine, University of Pennsylvania, Philadelphia, PA 19104, USA

12 Department of Clinical Sciences, College of Veterinary Medicine, Cornell University, Ithaca, NY 14853, USA

13 Institute for Animal Breeding and Genetics, University of Veterinary Medicine, Hannover, 30559 Hannover, Germany

14 Department of Clinical Sciences, Cummings School of Veterinary Medicine, Tufts University, Grafton, MA 01536, USA

15 Department of Animal Science, College of Agriculture and Life Sciences, Iowa State University, Ames, IA 50011, USA

16 College of Veterinary Medicine, North Carolina State University, Raleigh, NC 27607, USA

17 WALTHAM Centre for Pet Nutrition, Freeby Lane, Waltham on the Wolds, Leicestershire LE14 4RT, UK

18 Department of Psychiatry, University of Massachusetts Medical School, Worcester, MA 01655, USA

19 Department of Clinical Sciences, Faculty of Veterinary Medicine and Animal Science, Swedish University of Agricultural Sciences, SE-750 07 Uppsala, Sweden

20 School of Health and Sport Sciences, University of the Sunshine Coast, Sippy Downs, QLD 4558, Australia

21 Laboratory of Veterinary Radiology, Nippon Veterinary and Life Science University, Tokyo 180-8602, Japan

22 Sydney School of Veterinary Science, Faculty of Science, University of Sydney, Sydney, NSW 2006, Australia

23 Langford Vets, University of Bristol, Langford, Bristol BS40 5DU, UK

24 Pediatrics and Medical Genetics Service, College of Veterinary Medicine, Cornell University, Ithaca, NY 14853, USA

25 Department of Veterinary Biosciences; Department of Medical Genetics, University of Helsinki and Folkhälsan Research Center, 00014 Helsinki, Finland

26 Department of Medical Genetics, Center for Medical Genetics, Shinshu University Hospital, Matsumoto, Nagano 390-8621, Japan

27 SPF-Diana Pet food-Symrise Group-56250 Elven, France

28 Department of Veterinary Science, University of Kentucky - Lexington, Lexington, KY 40506, USA (In memoriam)

29 Vetsuisse Faculty, Institute of Genetics, University of Bern, 3001 Bern, Switzerland

30 Department of Surgical and Radiological Sciences, School of Veterinary Medicine, University of California Davis, One Shields Ave, Davis, CA 95616, USA

31 Dipartimento di Medicina Veterinaria, University of Milan, 20122 Milan, Italy 
32 Departments of Molecular Physiology and Biophysics, Cell and Developmental Biology, and Medicine, Vanderbilt University, School of Medicine, Nashville, TN 37232, USA

33 Centre for Veterinary Education, University of Sydney, Sydney, NSW 2006, Australia

34 Elanco Animal Health, Greenfield, IN 46140, USA

35 Department of Veterinary Integrative Biosciences, College of Veterinary Medicine, Texas A\&M University, College Station, TX 77845, USA

36 Department of Medicine and Epidemiology, School of Veterinary Medicine, University of California at Davis, Davis, CA 95616, USA

37 Department of Small Animal Clinical Sciences, Veterinary Medical Center, Michigan State University, East Lansing, MI 48824, USA

38 School of Veterinary Medicine, Faculty of Health \& Medical Sciences, Univesity of Surrey, Guildford, Surrey GU2 7AL, UK

39 Department of Ecology and Evolutionary Biology, University of California, Santa Cruz, Santa Cruz, CA 95064, USA

40 Center for Conservation and Research of Endangered Wildlife (CREW), Cincinnati Zoo \& Botanical Garden, Cincinnati, OH 45220, USA

41 Zoological Pathology Program, University of Illinois, Brookfield, IL 60513, USA

42 Division of Animal Sciences, College of Agriculture, Food and Natural Resources; School of Medicine, University of Missouri, Columbia, MO 65211, USA

43 Department of Biological Sciences, Bowling Green State University, Bowling Green, OH 43403, USA

Conflicts of Interest: Authors disclose no conflict of interest. The funding sponsors had no role in the design, execution, interpretation, or writing of the study. The authors may receive supportive funds from a genetic testing laboratory that would offer this variant as a commercialized test in the future.

\section{References}

1. MacKillop, E. Magnetic resonance imaging of intracranial malformations in dogs and cats. Vet. Radiol. Ultrasound. 2011, 52, S42-S51. [CrossRef] [PubMed]

2. Jurney, C.; Haddad, J.; Crawford, N.; Miller, A.D.; Van Winkle, T.J.; Vite, C.H.; Sponenberg, P.; Inzana, K.D.; Cook, C.R.; Britt, L.; et al. Polymicrogyria in standard poodles. J. Vet. Intern. Med. 2009, 23, 871-874. [CrossRef] [PubMed]

3. Goncalves, R.; Volk, H.; Smith, P.M.; Penderis, J.; Garosi, L.; MacKillop, E.; de Stefani, A.; Cherubini, G.; McConnell, J.F. Corpus callosal abnormalities in dogs. J. Vet. Intern. Med. 2014, 28, 1275-1279. [CrossRef] [PubMed]

4. Bernardino, F.; Rentmeister, K.; Schmidt, M.J.; Bruehschwein, A.; Matiasek, K.; Matiasek, L.A.; Lauda, A.; Schoon, H.A.; Fischer, A. Inferior cerebellar hypoplasia resembling a Dandy-Walker-like malformation in purebred Eurasier dogs with familial non-progressive ataxia: A retrospective and prospective clinical cohort study. PLoS ONE 2015, 10, e0117670. [CrossRef] [PubMed]

5. Gerber, M.; Fischer, A.; Jagannathan, V.; Drogemuller, M.; Drogemuller, C.; Schmidt, M.J.; Bernardino, F.; Manz, E.; Matiasek, K.; Rentmeister, K.; et al. A deletion in the VLDLR gene in Eurasier dogs with cerebellar hypoplasia resembling a Dandy-Walker-like malformation (DWLM). PLoS ONE 2015, 10, e0108917. [CrossRef]

6. Estey, C.M. Congenital Hydrocephalus. Vet. Clin. North. Am. Small Anim. Pract. 2016, 46, $217-229$. [CrossRef]

7. Selby, L.A.; Hayes, H.M., Jr.; Becker, S.V. Epizootiologic features of canine hydrocephalus. Am. J. Vet. Res. 1979, 40, 411-413.

8. Knowler, S.P.; Galea, G.L.; Rusbridge, C. Morphogenesis of Canine Chiari Malformation and Secondary Syringomyelia: Disorders of Cerebrospinal Fluid Circulation. Front. Vet. Sci. 2018, 5, 171. [CrossRef]

9. Schmidt, M.J.; Kampschulte, M.; Enderlein, S.; Gorgas, D.; Lang, J.; Ludewig, E.; Fischer, A.; Meyer-Lindenberg, A.; Schaubmar, A.R.; Failing, K.; et al. The Relationship between Brachycephalic Head Features in Modern Persian Cats and Dysmorphologies of the Skull and Internal Hydrocephalus. J. Vet. Intern. Med. 2017, 31, 1487-1501. [CrossRef]

10. Schlueter, C.; Budras, K.D.; Ludewig, E.; Mayrhofer, E.; Koenig, H.E.; Walter, A.; Oechtering, G.U. Brachycephalic feline noses: $\mathrm{CT}$ and anatomical study of the relationship between head conformation and the nasolacrimal drainage system. J. Feline Med. Surg. 2009, 11, 891-900. [CrossRef] 
11. Farnworth, M.J.; Chen, R.; Packer, R.M.; Caney, S.M.; Gunn-Moore, D.A. Flat feline faces: Is brachycephaly associated with respiratory abnormalities in the domestic cat (Felis catus)? PLoS ONE 2016, 11, e0161777. [CrossRef] [PubMed]

12. Mestrinho, L.A.; Louro, J.M.; Gordo, I.S.; Niza, M.; Requicha, J.F.; Force, J.G.; Gawor, J.P. Oral and dental anomalies in purebred, brachycephalic Persian and exotic cats. J. Am. Vet. Med. Assoc. 2018, 253, 66-72. [CrossRef] [PubMed]

13. Sponenberg, D.P.; Graf-Webster, E. Hereditary meningoencephalocele in Burmese cats. J. Hered. 1986, 77, 60. [CrossRef] [PubMed]

14. Lyons, L.A.; Erdman, C.A.; Grahn, R.A.; Hamilton, M.J.; Carter, M.J.; Helps, C.R.; Alhaddad, H.; Gandolfi, B. Aristaless-Like Homeobox protein 1 (ALX1) variant associated with craniofacial structure and frontonasal dysplasia in Burmese cats. Dev. Biol. 2016, 409, 451-458. [CrossRef] [PubMed]

15. Lowrie, M.; Wessmann, A.; Gunn-Moore, D.; Penderis, J. Quadrigeminal cyst management by cystoperitoneal shunt in a 4-year-old Persian cat. J. Feline Med. Surg. 2009, 11, 711-713. [CrossRef]

16. Reed, S.; Cho, D.Y.; Paulsen, D. Quadrigeminal arachnoid cysts in a kitten and a dog. J. Vet. Diagn. Invest. 2009, 21, 707-710. [CrossRef]

17. Herrmann, A.; Hecht, W.; Herden, C. Lissencephaly and microencephaly combined with hypoplasia of corpus callosum and cerebellum in a domestic cat. Tierarztl Prax Ausg K Kleintiere Heimtiere 2011, 39, 116-120.

18. Shimbo, G.; Tagawa, M.; Yanagawa, M.; Miyahara, K. MRI of lobar holoprosencephaly in a cat with hypodipsic hypernatraemia. JFMS Open Rep. 2018, 4. [CrossRef]

19. Boccanera, C.; Stabile, F.; Corvi, R.; Mariscoli, M.; Mandara, M.T. Hydrocephalus, supratentorial diverticulum and agenesis of the interthalamic adhesion and corpus callosum in a cat: MRI findings, treatment and follow-up. Vet. Record Case Rep. 2018, 6, e000416. [CrossRef]

20. Woerde, D.J.; Hoffmann, K.L.; Brown, N.L. Frontoethmoidal encephalocele in a cat. JFMS Open Rep. $2018,4$. [CrossRef]

21. Keating, M.K.; Sturges, B.K.; Siso, S.; Wisner, E.R.; Creighton, E.K.; Lyons, L.A. Characterization of an Inherited Neurologic Syndrome in Toyger Cats with Forebrain Commissural Malformations, Ventriculomegaly and Interhemispheric Cysts. J. Vet. Intern. Med. 2016, 30, 617-626. [CrossRef] [PubMed]

22. Gandolfi, B.; Alhaddad, H.; Abdi, M.; Bach, L.H.; Creighton, E.K.; Davis, B.W.; Decker, J.E.; Dodman, N.H.; Ginns, E.I.; Grahn, J.C.; et al. Applications and efficiencies of the first cat 63K DNA array. Sci Rep. 2018, 8, 7024. [CrossRef] [PubMed]

23. Lipinski, M.J.; Amigues, Y.; Blasi, M.; Broad, T.E.; Cherbonnel, C.; Cho, G.J.; Corley, S.; Daftari, P.; Delattre, D.R.; Dileanis, S.; et al. An international parentage and identification panel for the domestic cat (Felis catus). Anim Genet. 2007, 38, 371-377. [CrossRef] [PubMed]

24. Jones, O.R.; Wang, J. COLONY: A program for parentage and sibship inference from multilocus genotype data. Mol. Ecol. Resour. 2010, 10, 551-555. [CrossRef]

25. Wang, J. A simulation module in the computer program COLONY for sibship and parentage analysis. Mol. Ecol. Resour. 2013, 13, 734-739. [CrossRef]

26. Mullikin, J.C.; Hansen, N.F.; Shen, L.; Ebling, H.; Donahue, W.F.; Tao, W.; Saranga, D.J.; Brand, A.; Rubenfield, M.J.; Young, A.C.; et al. Light whole genome sequence for SNP discovery across domestic cat breeds. BMC Genom. 2010, 11, 406. [CrossRef]

27. Buckley, R.M.; Davis, B.W.; Brashear, W.A.; Farias, F.H.G.; Kuroki, K.; Graves, T.; Hillier, L.W.; Kremitzki, M.; Li, G.; Middleton, R.; et al. A new domestic cat genome assembly based on long sequence reads empowers feline genomic medicine and identifies a novel gene for dwarfism. bioRxiv 2020. [CrossRef]

28. Purcell, S.; Neale, B.; Todd-Brown, K.; Thomas, L.; Ferreira, M.A.; Bender, D.; Maller, J.; Sklar, P.; de Bakker, P.I.; Daly, M.J.; et al. PLINK: A tool set for whole-genome association and population-based linkage analyses. Am. J. Hum. Genet. 2007, 81, 559-575. [CrossRef]

29. Spielman, R.S.; Ewens, W.J. A sibship test for linkage in the presence of association: The sib transmission/disequilibrium test. Am. J. Hum. Genet. 1998, 62, 450-458. [CrossRef]

30. Barrett, J.C.; Fry, B.; Maller, J.; Daly, M.J. Haploview: Analysis and visualization of LD and haplotype maps. Bioinformatics 2005, 21, 263-265. [CrossRef]

31. Lyons, L.A.; Creighton, E.K.; Alhaddad, H.; Beale, H.C.; Grahn, R.A.; Rah, H.; Maggs, D.J.; Helps, C.R.; Gandolfi, B. Whole genome sequencing in cats, identifies new models for blindness in AIPL1 and somite segmentation in HES7. BMC Genom. 2016, 17, 265. [CrossRef] [PubMed] 
32. Li, H.; Durbin, R. Fast and accurate short read alignment with Burrows-Wheeler transform. Bioinformatics 2009, 25, 1754-1760. [CrossRef] [PubMed]

33. McKenna, A.; Hanna, M.; Banks, E.; Sivachenko, A.; Cibulskis, K.; Kernytsky, A.; Garimella, K.; Altshuler, D.; Gabriel, S.; Daly, M.; et al. The Genome Analysis Toolkit: A MapReduce framework for analyzing next-generation DNA sequencing data. Genome Res. 2010, 20, 1297-1303. [CrossRef] [PubMed]

34. Van der Auwera, G.A.; Carneiro, M.O.; Hartl, C.; Poplin, R.; Del Angel, G.; Levy-Moonshine, A.; Jordan, T.; Shakir, K.; Roazen, D.; Thibault, J.; et al. From FastQ data to high confidence variant calls: The Genome Analysis Toolkit best practices pipeline. Curr. Protoc. Bioinform. 2013, 43, 11.10.1-11.10.33. [CrossRef]

35. Thorvaldsdottir, H.; Robinson, J.T.; Mesirov, J.P. Integrative Genomics Viewer (IGV): High-performance genomics data visualization and exploration. Brief. Bioinform. 2013, 14, 178-192. [CrossRef]

36. Toonen, R.J.; Hughes, S. Increased throughput for fragment analysis on an ABI PRISM 377 automated sequencer using a membrane comb and STRand software. Biotechniques 2001, 31, 1320-1324. [PubMed]

37. Buckley, R.M.; Gandolfi, B.; Creighton, E.K.; Pyne, C.A.; Leroy, M.L.; Senter, D.A.; Bouhan, D.M.; Gobble, J.R.; Abitbol, M.; Lyons, L.A.; et al. Werewolf, there wolf: Variants in Hairless associated wih hypotrichia and roaning in the lykoi cat breed. bioRxiv 2020. [CrossRef]

38. Ancot, F.; Lemay, P.; Knowler, S.P.; Kennedy, K.; Griffiths, S.; Cherubini, G.B.; Sykes, J.; Mandigers, P.J.J.; Rouleau, G.A.; Rusbridge, C.; et al. A genome-wide association study identifies candidate loci associated to syringomyelia secondary to Chiari-like malformation in Cavalier King Charles Spaniels. BMC Genet. 2018, 19, 16. [CrossRef] [PubMed]

39. Marchant, T.W.; Dietschi, E.; Rytz, U.; Schawalder, P.; Jagannathan, V.; Hadji Rasouliha, S.; Gurtner, C.; Waldvogel, A.S.; Harrington, R.S.; Drogemuller, M.; et al. An ADAMTS3 missense variant is associated with Norwich Terrier upper airway syndrome. PLoS Genet. 2019, 15, e1008102. [CrossRef] [PubMed]

40. Aberdein, D.; Munday, J.S.; Gandolfi, B.; Dittmer, K.E.; Malik, R.; Garrick, D.J.; Lyons, L.A.; Lives, C. A FAS-ligand variant associated with autoimmune lymphoproliferative syndrome in cats. Mamm Genome 2017, 28, 47-55. [CrossRef] [PubMed]

41. Gandolfi, B.; Grahn, R.A.; Creighton, E.K.; Williams, D.C.; Dickinson, P.J.; Sturges, B.K.; Guo, L.T.; Shelton, G.D.; Leegwater, P.A.; Longeri, M.; et al. COLQ variant associated with Devon Rex and Sphynx feline hereditary myopathy. Anim Genet. 2015, 46, 711-715. [CrossRef] [PubMed]

42. Mauler, D.A.; Gandolfi, B.; Reinero, C.R.; O’Brien, D.P.; Spooner, J.L.; Lyons, L.A.; 99 Lives Consortium. Precision Medicine in Cats: Novel Niemann-Pick Type C1 Diagnosed by Whole-Genome Sequencing. J. Vet. Intern. Med. 2017, 31, 539-544. [CrossRef] [PubMed]

43. Oh, A.; Pearce, J.W.; Gandolfi, B.; Creighton, E.K.; Suedmeyer, W.K.; Selig, M.; Bosiack, A.P.; Castaner, L.J.; Whiting, R.E.; Belknap, E.B.; et al. Early-Onset Progressive Retinal Atrophy Associated with an IQCB1 Variant in African Black-Footed Cats (Felis nigripes). Sci. Rep. 2017, 7, 43918. [CrossRef] [PubMed]

44. Ontiveros, E.S.; Ueda, Y.; Harris, S.P.; Stern, J.A.; Lives, C. Precision medicine validation: Identifying the MYBPC3 A31P variant with whole-genome sequencing in two Maine Coon cats with hypertrophic cardiomyopathy. J. Feline Med. Surg. 2019, 21, 1086-1093. [CrossRef]

45. Jaffey, J.A.; Reading, N.S.; Giger, U.; Abdulmalik, O.; Buckley, R.M.; Johnstone, S.; Lyons, L.A.; Lives Cat Genome, C. Clinical, metabolic, and genetic characterization of hereditary methemoglobinemia caused by cytochrome b5 reductase deficiency in cats. J. Vet. Intern. Med. 2019, 33, 2725-2731. [CrossRef]

46. Buckley, R.M.; Grahn, R.A.; Gandolfi, B.; Herrick, J.R.; Kittleson, M.D.; Bateman, H.L.; Newsom, J.; Swanson, W.F.; Prieur, D.J.; Lyons, L.A. Assisted reproduction mediated resurrection of a feline model for Chediak-Higashi syndrome caused by a large duplication in LYST. Sci. Rep. 2020, 10, 64. [CrossRef]

47. Summers, A.D.; Reefhuis, J.; Taliano, J.; Rasmussen, S.A. Nongenetic risk factors for holoprosencephaly: An updated review of the epidemiologic literature. Am. J. Med. Genet. C Semin Med. Genet. 2018, 178, 151-164. [CrossRef]

48. Dubourg, C.; Kim, A.; Watrin, E.; de Tayrac, M.; Odent, S.; David, V.; Dupe, V. Recent advances in understanding inheritance of holoprosencephaly. Am. J. Med. Genet. C Semin Med. Genet. 2018, 178, 258-269. [CrossRef]

49. Kruszka, P.; Martinez, A.F.; Muenke, M. Molecular testing in holoprosencephaly. Am. J. Med. Genet. C Semin Med. Genet. 2018, 178, 187-193. [CrossRef]

50. Roessler, E.; Hu, P.; Muenke, M. Holoprosencephaly in the genomics era. Am. J. Med. Genet. C Semin Med. Genet. 2018, 178, 165-174. [CrossRef] 
51. Lee, K.J.; Mendelsohn, M.; Jessell, T.M. Neuronal patterning by BMPs: A requirement for GDF7 in the generation of a discrete class of commissural interneurons in the mouse spinal cord. Genes Dev. 1998, 12, 3394-3407. [CrossRef] [PubMed]

52. Heussler, H.S.; Suri, M.; Young, I.D.; Muenke, M. Extreme variability of expression of a Sonic Hedgehog mutation: Attention difficulties and holoprosencephaly. Arch. Dis Child. 2002, 86, 293-296. [CrossRef] [PubMed]

53. Marini, M.; Cusano, R.; De Biasio, P.; Caroli, F.; Lerone, M.; Silengo, M.; Ravazzolo, R.; Seri, M.; Camera, G. Previously undescribed nonsense mutation in SHH caused autosomal dominant holoprosencephaly with wide intrafamilial variability. Am. J. Med. Genet. A 2003, 117A, 112-115. [CrossRef] [PubMed]

54. Hehr, U.; Gross, C.; Diebold, U.; Wahl, D.; Beudt, U.; Heidemann, P.; Hehr, A.; Mueller, D. Wide phenotypic variability in families with holoprosencephaly and a sonic hedgehog mutation. Eur. J. Pediatr. 2004, 163, 347-352. [CrossRef]

55. Hogan, B.L. Bone morphogenetic proteins: Multifunctional regulators of vertebrate development. Genes Dev. 1996, 10, 1580-1594. [CrossRef]

56. Mehler, M.F.; Mabie, P.C.; Zhang, D.; Kessler, J.A. Bone morphogenetic proteins in the nervous system. Trends Neurosci. 1997, 20, 309-317. [CrossRef]

57. Wang, R.N.; Green, J.; Wang, Z.; Deng, Y.; Qiao, M.; Peabody, M.; Zhang, Q.; Ye, J.; Yan, Z.; Denduluri, S.; et al. Bone Morphogenetic Protein (BMP) signaling in development and human diseases. Genes Dis 2014, 1, 87-105. [CrossRef]

58. Mikic, B.; Bierwert, L.; Tsou, D. Achilles tendon characterization in GDF-7 deficient mice. J. Orthop Res. 2006, 24, 831-841. [CrossRef]

59. Mikic, B.; Ferreira, M.P.; Battaglia, T.C.; Hunziker, E.B. Accelerated hypertrophic chondrocyte kinetics in GDF-7 deficient murine tibial growth plates. J. Orthop Res. 2008, 26, 986-990. [CrossRef]

60. Settle, S.; Marker, P.; Gurley, K.; Sinha, A.; Thacker, A.; Wang, Y.; Higgins, K.; Cunha, G.; Kingsley, D.M. The BMP family member Gdf7 is required for seminal vesicle growth, branching morphogenesis, and cytodifferentiation. Dev. Biol. 2001, 234, 138-150. [CrossRef]

61. Maloul, A.; Rossmeier, K.; Mikic, B.; Pogue, V.; Battaglia, T. Geometric and material contributions to whole bone structural behavior in GDF-7-deficient mice. Connect. Tissue Res. 2006, 47, 157-162. [CrossRef] [PubMed]

62. Palles, C.; Chegwidden, L.; Li, X.; Findlay, J.M.; Farnham, G.; Castro Giner, F.; Peppelenbosch, M.P.; Kovac, M.; Adams, C.L.; Prenen, H.; et al. Polymorphisms near TBX5 and GDF7 are associated with increased risk for Barrett's esophagus. Gastroenterology 2015, 148, 367-378. [CrossRef] [PubMed]

63. Becker, J.; May, A.; Gerges, C.; Anders, M.; Schmidt, C.; Veits, L.; Noder, T.; Mayershofer, R.; Kreuser, N.; Manner, H.; et al. The Barrett-associated variants at GDF7 and TBX5 also increase esophageal adenocarcinoma risk. Cancer Med. 2016, 5, 888-891. [CrossRef] [PubMed]

(C) 2020 by the authors. Licensee MDPI, Basel, Switzerland. This article is an open access article distributed under the terms and conditions of the Creative Commons Attribution (CC BY) license (http://creativecommons.org/licenses/by/4.0/). 